Bull. Austral. Math. Soc.

$20 \mathrm{D} 60,20 \mathrm{~F} 99$

Vol. 46 (1992) [263-269]

\title{
THE CLASSIFICATION OF GROUPS WITH THE SMALL SQUARING PROPERTY ON 3-SETS
}

\author{
P. LONGOBARDI AND M. MaJ
}

Let $G$ be a group and $k$ an integer greater than 1 . We say that $G$ has the square property of $k$-sets and we write $G \in D S(k)$ if $\left|X^{2}\right|<k^{2}$ for any subset $X$ of $G$ of order $k$. The groups in $D S(2)$ are exactly the Dedekind groups, as Freiman showed. The class $D S(3)$ has been recently studied by Berkovich, Freiman and Praeger. In this paper we complete the classification of $D S(3)$-groups by characterising finite 2-groups of exponent 4 in $D S(3)$.

\section{INTRODUCTION}

Let $G$ be a group and $k$ an integer greater than 1 .

Following [1] we say that $G$ has the square property of $k$-sets and we write $G \in$ $D S(k)$ if $\left|X^{2}\right|<k^{2}$ for any subset $X$ of $G$ of order $k$.

The groups in $D S(2)$ are exactly the Dedekind groups, as Freiman showed in [2]. The class $D S(3)$ has been recently studied by Berkovich, Freiman and Praeger. They came close to a classification of the finite $D S(3)$-groups. There was only one case left, the case where $G$ is a finite 2 -group of exponent 4 .

In this paper we complete the classification of $D S(3)$-groups. We prove the following:

Theorem A. Let $G$ be a finite 2-group of exponent 4. Then $G \in D S(3)$ if and only if one of the following holds:

(1) $G$ is abelian,

(2) $\mho_{1}(G)=\left\langle x^{2} / x \in G\right\rangle$ has order 2,

(3) $G=A\langle x\rangle$, where $A$ is abelian, $x^{2} \in A, a^{x}=a^{-1}$ for every $a \in A$,

(4) $G=D \times\langle a, b, c\rangle$, where $D$ is an elementary abelian 2-group, $|a|=|b|=$ $|c|=4, a^{b}=a^{-1},[a, c]=[b, c]=1, c^{2}=a^{2} b^{2}$.

(5) $G=D \times\langle a, b, c, d\rangle$, where $D$ is an elementary abelian 2-group, $a^{b}=$ $a^{-1}, c^{b}=c^{-1}, c^{d}=c^{-1},[a, c]=[a, d]=[b, d]=1,|a|=|b|=|c|=$ $|d|=4, c^{2}=d^{2}=a^{2} b^{2}$.

Theorem A together with the results of [1] gives the following:

Received 12 November 1991

Copyright Clearance Centre, Inc. Serial-fee code: 0004-9729/92 \$A2.00+0.00. 
Theorem B. Let $G$ be a finite group. Then $G \in D S(3)$ if and only if $G$ is abelian, or $G=A\langle x\rangle$, where $A$ is abelian, $x^{2} \in A, a^{x}=a^{-1}$, for any $a \in A$, or $G$ is a 2-group of exponent 4 satisfying (2) or (4) or (5) of Theorem $A$.

It is not difficult to show that in fact Theorem $B$ holds for any group (not necessarily finite) as we prove in Section 3.

Our notation is the usual one (see for instance [4]).

If $G$ is a finite 2-group we put

$$
\Omega_{1}(G)=\langle x \in G /|x|=2\rangle, \quad \mho_{1}(G)=\left\langle x^{2} / x \in G\right\rangle
$$

\section{Finite 2-groups of EXPONENT 4 in $D S(3)$}

LEMMA 2.1. Let $G \in D S(3)$ be a finite 2-group of exponent 4. Then $G / Z(G)$ has exponent 2. In particular $G$ is nilpotent of class at most 2.

Proof: We prove that $x^{2} \in Z(G)$ for every $x \in G$. Write $\Phi(G)$ for the Frattini subgroup of $G$. Then it suffices to show that $x^{2} y=y x^{2}$ for every $y \in G-\Phi(G)$ such that $x \Phi(G) \neq y \Phi(G)$. Assume by contradiction that there exist $x, y \in G$ with $y \Phi(G) \neq \Phi(G), x^{2} y \neq y x^{2}$ and $x \Phi(G) \neq y \Phi(G)$. If $|y|=2$, then $\left|x^{2} y\right|=4$; hence we may assume, replacing $y$ with $x^{2} y$ if necessary, that $|y|=4$.

For any $g \in G-\left\langle x^{2}, y\right\rangle$ consider the set $X=\left\{x^{2}, y, g\right\}$. Then from $\left|X^{2}\right|<9$ it follows that $g \in C_{G}\left(x^{2}\right) \cup C_{G}(y)$ or $g^{2}=y^{2}$ or $g^{2}=1$ (from $g^{2}=x^{2} y$ or $g^{2}=y x^{2}$ we get $y \in \Phi(G)$, a contradiction).

In particular either $(x y)^{2}=1$ or $(x y)^{2}=y^{2}$ since $\left[x y, x^{2}\right] \neq 1$ and $[x y, y] \neq 1$. But $(x y)^{2}=y^{2}$ implies $x y x y=y^{2}, y^{-1} x y=x^{-1}$ and $x^{2} y=y x^{2}$, a contradiction. Therefore $(x y)^{2}=1$; hence $\left\langle x^{2}, x y\right\rangle \cong D_{4}$ and $x^{2} x y=x^{-1} y$ has order 4 . Thus, as before, from $x^{-1} y \notin C_{G}\left(x^{2}\right) \cup C_{G}(y)$ it follows that $\left(x^{-1} y\right)^{2}=y^{2}$, and hence $x^{-1} y x^{-1} y=y^{2}, y^{-1} x^{-1} y=x$ and $x^{2} y=y x^{2}$, again a contradiction.

In the following $G$ will always be a finite 2-group of exponent 4 . We apply Lemma 2.1 to get the following useful result:

LEMMA 2.2. Let $G \in D S(3)$ and $x, y$ be elements of $G$ such that $[x, y] \neq 1$ and $x^{2} \neq y^{2}$. Then for every $g \in G$ we have $g \in C_{G}(x) \cup C_{G}(y)$ or $g^{2}=x^{2}$ or $g^{2}=y^{2}$.

Proof: The result follows easily from $\left|\{x, y, g\}^{2}\right|<9$, using the fact that $G^{\prime} \leqslant$ $Z(G)$ and $g^{2} \in Z(G)$.

The following lemma gives some more precise information.

LеммA 2.3. Let $G \in D S(3)$ and $x, y$ be elements of $G$ such that $[x, y] \neq 1$ and $x^{2} \neq y^{2}$. Assume $(x y)^{2}=y^{2}$. Then for every $g \in G$ we have $g \in C_{G}(x)$ or $g^{2}=x^{2}$ or $g^{2}=y^{2}$. 
Proof: Let $g \in G$. Then, by Lemma 2.2, $g \in C_{G}(x) \cup C_{G}(y)$ or $g^{2}=x^{2}$ or $g^{2}=y^{2}$. Similarly from $(x y)^{2}=y^{2}$ it follows, by Lemma 2.2, that $g \in C_{G}(x) \cup C_{G}(x y)$ or $g^{2}=x^{2}$ or $g^{2}=y^{2}$. If $g \in C_{G}(y) \cap C_{G}(x y)$, then $g \in C_{G}(x)$. Hence $g \in C_{G}(x)$ or $g^{2}=x^{2}$ or $g^{2}=y^{2}$, as required.

Let $G$ be a finite non-abelian group in $D S(3)$ of exponent 4 . If $\langle x \in G /|x|=4\rangle$ is a proper subgroup of $G$, then $G$ is a $D$-group with the notation of [1]. Then, by the results of [1], (3) of Theorem A holds.

Thus in the following we assume $G=\langle x \in G /|x|=4\rangle$.

First we remark:

Lemma 2.4. Let $a, b \in G$ be such that $a^{2} \neq b^{2}, a^{b}=a^{-1},|a|=4$. Then $\langle a\rangle$ is normal in $G$.

Proof: First we prove that $[a, g] \in\left\{a^{2}, b^{2}, 1\right\}$ for every $g \in G$. Let $g \in G-$ $C_{G}(a)$. Then by Lemma 2.3 either $g^{2}=a^{2}$ or $g^{2}=b^{2}$, and similarly either $(a g)^{2}=a^{2}$ or $(a g)^{2}=b^{2}$. Furthermore we have $(a g)^{2}=a^{2} g^{2}[a, g]$. If $g^{2}=a^{2}$, then $[a, g]=$ $(a g)^{2} \in\left\{a^{2}, b^{2}\right\}$. If $g^{2}=b^{2}$, then $[a, g]=(a g)^{2} a^{2} b^{2}$ and $[a, g]=b^{2}$ if $(a g)^{2}=a^{2}$, while $[a, g]=a^{2}$ if $(a g)^{2}=b^{2}$. Now, if $|b|=2$ then $[a, g]$ is always in $\langle a\rangle$, and $\langle a\rangle$ is normal in $G$, as required. If $|b|=4$ and $[a, g]=b^{2}$ for some $g \in G$, then $[a, b g]=a^{2} b^{2} \notin\left\{1, a^{2}, b^{2}\right\}$, a contradiction.

Now we study groups satisfying our conditions and with $\Omega_{1}(G) \nsubseteq Z(G)$.

2.5. Assume there exists $g \in G$ with $|g|=2, g \notin Z(G)$. Then $\left|\mho_{1}(G)\right|=2$ and (2) of Theorem $A$ holds.

Proof: Let $g \notin Z(G)$, with $|g|=2$. Then there exists $a \in G$, with $|a|=4$, $a g \neq g a$, since $G=\langle x \in G /|x|=4\rangle$. By Lemma 2.2 either $(a g)^{2}=a^{2}$ or $(a g)^{2}=1$. From $(a g)^{2}=a^{2}$ it follows that agag $=a^{2}$, whence $a g=g a$, a contradiction. Hence $(a g)^{2}=1$, and $g^{-1} a g=a^{-1}$. Thus by Lemma 2.4 we have $\langle a\rangle$ normal in $G$. Moreover for every $x \in G,|x|=4$, either $a^{2}=x^{2}$ or $x \in C_{G}(a)$ by Lemma 2.3. There exists $b \in G$, with $|b|=4$ and $a b \neq b a$ since $a \notin Z(G)$. Then $b^{2}=a^{2}$ and $|a b|=4$. If $[b, g]=1$, then $[a b, g] \neq 1$. Without loss of generality we may assume $[b, g] \neq 1$. Then, arguing on $b$ as previously on $a$, we get $\langle b\rangle$ normal in $G$ and either $x^{2}=b^{2}=a^{2}$ or $x \in C_{G}(b)$ for every $x \in G,|x|=4$.

Now assume by contradiction that there is an element $x \in G$, with $x^{2} \neq a^{2}$ and $|x|=4$. Thus $x \in C_{G}(a)$ and $x \in C_{G}(b)$. But then $a x \notin C_{G}(b)$ and $|a x|=4$, hence $(a x)^{2}=b^{2}=a^{2}$ and $x^{2}=1$, a contradiction.

It will be shown later that groups satisfying (1) to (5) of Theorem A have the property $D S(3)$.

Now we assume $\Omega_{1}(G) \leqslant Z(G)$. We start with two particular cases. 
CASE 2.6. Let $a, b \in G$ be such that $|a|=|b|=4, a^{2} \neq b^{2}$ and $a^{b}=a^{-1}$. Assume $G=\langle a, b\rangle\left(C_{G}(a) \cap C_{G}(b)\right)$ and that (3) does not hold. Then (4) of Theorem A holds.

Proof: First we remark that $y^{2} \in\left\langle a^{2} b^{2}\right\rangle$ for every $y \in C_{G}(a) \cap C_{G}(b)$. In fact let $y \in C_{G}(a) \cap C_{G}(b)$. Then $y b \notin C_{G}(a)$ and, by Lemma 2.3, either $(y b)^{2}=a^{2}$ or $(y b)^{2}=b^{2}$. If $(y b)^{2}=a^{2}$, then $y^{2} b^{2}=a^{2}$ and $y^{2}=a^{2} b^{2}$, while if $y^{2} b^{2}=(y b)^{2}=b^{2}$, then $y^{2}=1$.

Now we prove that $C_{G}(a) \cap C_{G}(b)$ is abelian. Let $y_{1}, y_{2} \in C_{G}(a) \cap C_{G}(b)$ and, by contradiction, assume $\left[y_{1}, y_{2}\right] \neq 1$. Then $\left|y_{1}\right|=\left|y_{2}\right|=4$, since $\Omega_{1}(G) \leqslant Z(G)$. The elements $a y_{1}, b y_{1}$ do not permute, and $\left(a y_{1}\right)^{2}=a^{2} y_{1}^{2} \neq b^{2} y_{1}^{2}=\left(b y_{1}\right)^{2}$. Then Lemma 2.2 applies and $y_{2} \in C_{G}\left(a y_{1}\right) \cup C_{G}\left(b y_{2}\right)$ or $y_{2}^{2}=\left(a y_{1}\right)^{2}=a^{2} y_{1}^{2}$ or $y_{2}^{2}=\left(b y_{1}\right)^{2}=b^{2} y_{1}^{2}$. But $y_{2} \notin C_{G}\left(y_{1}\right)$ and $y_{2}^{2}=y_{1}^{2}$, a contradiction. Therefore $C_{G}(a) \cap C_{G}(b)$ is an abelian group of exponent 4 and with $\mho_{1}\left(C_{G}(a) \cap C_{G}(b)\right)=\left\langle a^{2} b^{2}\right\rangle$. Thus $C_{G}(a) \cap C_{G}(b)=$ $D \times\langle c\rangle$, where $\exp D=2$ and $c^{2}=a^{2} b^{2}$, and (4) of Theorem A holds.

CASE 2.7. Assume that there exist $a, b, c, d \in G$ such that $|a|=|b|=|c|=$ $|d|=4$, and

(1) $\langle a, c\rangle=\langle a\rangle \times\langle c\rangle$,

(2) $a^{b}=a^{-1}, c^{b}=c^{-1}, b^{2} \neq a^{2}, c^{2}$,

(3) $a^{d}=a, b^{d}=b, c^{d}=c^{-1}$.

Then $c^{2}=d^{2}=a^{2} b^{2}$, and (5) of Theorem $A$ holds.

Proof: First we prove that $c^{2}=d^{2}=a^{2} b^{2}$. From $b d \notin C_{G}(a)$, it follows that either $(b d)^{2}=a^{2}$ or $(b d)^{2}=b^{2}$, by Lemma 2.3. If $(b d)^{2}=b^{2}$ then we have $d^{2}=1$ which is a contradiction. Then $b^{2} d^{2}=a^{2}$ and $d^{2}=a^{2} b^{2}$. Similarly if $c^{2} \neq d^{2}$, from $b c \notin C_{G}(c) \cup C_{G}(d)$ it follows, by Lemma 2.2 , that either $(b c)^{2}=c^{2}$ or $(b c)^{2}=d^{2}$. But $(b c)^{2}=b^{2}$, and so either $b^{2}=c^{2}$ or $b^{2}=d^{2}$, a contradiction.

Therefore $c^{2}=d^{2}=a^{2} b^{2}$.

Now write $D=C_{G}(a) \cap C_{G}(c)$. Then we have $D$ normal in $G$ and $|G / D| \leqslant$ 4 , since $\langle a\rangle,\langle c\rangle$ are normal in $G$ by Lemma 2.4. Moreover $G=D\langle b, d\rangle$ because $b, d, b d \notin C_{G}(a) \cap C_{G}(c)$.

We prove that $g^{2} \in\left\{1, a^{2}, b^{2}, a^{2} b^{2}\right\}$ for every $g \in D$. From that it follows that one of $g, g a, g c a, g c$ has order 2 and $D=\Omega_{1}(D)\langle a, c\rangle:$ thus $D=\langle a\rangle \times\langle c\rangle \times Y$ where $Y \leqslant \Omega_{1}(D) \leqslant Z(G)$, and $G$ has the required structure.

Assume by contradiction that there exists $g \in D$ with $g^{2} \notin\left\{1, a^{2}, b^{2}, a^{2} b^{2}\right\}$. If $g$ commutes with $b$, then from $b g \notin C_{G}(a)$ it follows by Lemma 2.3 that either $(b g)^{2}=b^{2} g^{2}=a^{2}$ or $b^{2} g^{2}=(b g)^{2}=b^{2}$, a contradiction. Similarly if $g \in C_{G}(d)$, then from $d g \notin C_{G}(c)$ it follows that either $d^{2} g^{2}=(d g)^{2}=c^{2}$ or $d^{2} g^{2}=(d g)^{2}=b^{2}$, a contradiction. Thus $g \notin C_{G}(b) \cup C_{G}(d)$. Now we have $g^{2} \neq d^{2}=c^{2}$. By Lemma 2.2, 
every element $x \in G$ is either in $C_{G}(g) \cup C_{G}(d)$ or is such that $x^{2}=g^{2}$ or $x^{2}=d^{2}$. Then $(c b)^{2}=g^{2}$ or $(c b)^{2}=d^{2}$. But $(c b)^{2}=b^{2}$ and we get a contradiction.

Now we can complete our characterisation of finite groups of exponent 4 in $D S(3)$.

Proof of Theorem A: Let $G \in D S(3)$ be a finite 2-group of exponent 4 . Assume $G$ non-abelian and $\left|\mho_{1}(G)\right|>2$. Also suppose that (3) does not hold. Then every element of order 2 is in the centre of $G$ by Lemma 2.5. Moreover there is an element $x \in G$ with $|x|=4$ and $\langle x\rangle$ is not normal in $G$, since $G$ is not a Dedekind group. Thus there exists $y \in G$ of order 4 with $y \notin N_{G}(\langle x\rangle)$. If $(x y)^{2}=y^{2}$, then $y^{-1} x y=x^{-1}$ and $y \in N_{G}(\langle x\rangle)$, a contradiction. Hence $(x y)^{2} \neq y^{2}$. Moreover $|x y|=4$, because $x y \notin C_{G}(x)$. Therefore we have found two elements $a, b \in G$ with $a b \neq b a$, $a^{2} \neq b^{2},|a|=|b|=4$. By Lemma 2.2 we have either $(a b)^{2}=a^{2}$ or $(a b)^{2}=b^{2}$. Without loss of generality we can assume $(a b)^{2}=b^{2}$. Then $b^{-1} a b=a^{-1}$, and $\langle a\rangle$ is normal in $G$ by Lemma 2.4. Moreover, for every $g \in G-C_{G}(a)$ we have either $g^{2}=a^{2}$ or $g^{2}=b^{2}$, by Lemma 2.3. Write $C=C_{G}(a)$. Then $C$ is normal in $G$ and $|G / C|=2$.

Assume first $C \leqslant C_{G}(b) \cup a^{-1} C_{G}(b)$; then $G=\langle a, b\rangle\left(C \cap C_{G}(b)\right)$. Now 2.6 applies and (4) holds. Then we can assume that there exists $c \in C-\left(C_{G}(b) \cup a^{-1} C_{G}(b)\right)$. Thus $|a c|>2$ and $a^{2} \neq c^{2}$. We claim that $c^{b}=c^{-1}$. In fact from $b c \notin C_{G}(a)$ it follows that either $(b c)^{2}=a^{2}$ or $(b c)^{2}=b^{2}$. If $(b c)^{2}=b^{2}$, then $c^{b}=c^{-1}$. Assume by contradiction $(b c)^{2}=a^{2}$. If $b^{2}=c^{2}$, then $a^{2}=(b c)^{2}=b^{2} c^{2}[b, c]$, thus $[b, c]=a^{2}=[b, a]$ and $[b, a c]=1$, a contradiction. If $b^{2} \neq c^{2}$, then from $[b, c] \neq 1$ it follows that either $(b c)^{2}=b^{2}$ or $(b c)^{2}=c^{2}$ by Lemma 2.2. But $(b c)^{2}=a^{2}$ and $a^{2} \neq b^{2}, c^{2}$ and we have a contradiction. Therefore we get $c^{b}=c^{-1}$. Moreover, replacing $c$ by $c a$ if necessary, we can assume $c^{2} \notin\left\{1, a^{2}, b^{2}\right\}$. Then for every $g \in G-C_{G}(c)$ either $g^{2}=b^{2}$ or $g^{2}=c^{2}$ by Lemma 2.3 .

Now we have $G=C\langle b\rangle$. If $d^{b}=d^{-1}$ for every $d \in C$, then $C$ is abelian and (3) holds. Assume that there exists $d \in C$ such that $d^{b} \neq d^{-1}$. Then $(d b)^{2} \neq b^{2}$ and $(d b)^{2}=a^{2}$ since $d b \notin C_{G}(a)$. Similarly from $d b \notin C_{G}(c)$ it follows that either $(d b)^{2}=c^{2}$ or $(d b)^{2}=b^{2}$. But $b^{2}, c^{2}$ are different from $a^{2}$. Hence $d b \in C_{G}(c)$ and $c^{d}=c^{-1}$. Analogously we get that either $d^{2}=c^{2}$ or $d^{2}=b^{2}$ (since $[c, d] \neq 1$ ).

First assume $d^{2}=c^{2}$. Consider the elements $a c, d b$. Then we have $[a c, d b]=$ $[a, b] \neq 1,(a c)^{2}=a^{2} c^{2} \neq(d b)^{2}=a^{2}$. Hence by Lemma $2.2 c d \in C_{G}(a c) \cup C_{G}(d b)$ or $(c d)^{2}=(a c)^{2}=a^{2} c^{2}$ or $(c d)^{2}=a^{2}$. But $(c d)^{2}=d^{2}$, and then the only possibility is $[c d, d b]=[d, b]=1$. Now the elements $a, b, c, d$ satisfy the hypothesis of 2.7 and (5) holds.

Now assume $d^{2}=b^{2}$. Then from $a^{2}=(b d)^{2}=b^{2} d^{2}[d, b]$ it follows that $[d, b]=$ $a^{2}=[a, b]$. Hence $[a d, b]=1$. Thus, with $d^{\prime}=a d$, we have $a^{b}=a^{-1}, a^{c}=a, a^{d^{\prime}}=a$, $c^{d^{\prime}}=c^{d}=c^{-1}, b^{d^{\prime}}=b$ and the elements $a, b, c, d^{\prime}$ satisfy the hypothesis of 2.7 and again (5) holds. 
Conversely assume that one of (1), (2), (3), (4), (5) holds.

If (1) or (2) or (3) holds, trivially $G \in D S(3)$.

Now assume that (4) holds. Then $\Omega_{1}(G) \leqslant Z(G)$ and $\mho_{1}(G)=\left\{1, a^{2}, b^{2}, c^{2}\right\}$. Furthermore every element $x \in G$ can be written in the form $x=d a^{\alpha} b^{\beta} c^{\gamma}$, where $d \in \Omega_{1}(G), \alpha, \beta, \gamma \equiv 0,1(\bmod 2)$. Then we have $\left(a^{\alpha} b c^{\gamma}\right)^{2}=a^{\alpha} b c^{\gamma} a^{\alpha} b c^{\gamma}=b^{2} c^{2 \gamma} \in$ $\left\{a^{2}, b^{2}\right\}$ and $\left(a^{\alpha} c^{\gamma}\right)^{2}=c^{2}$ if and only if $\alpha \equiv 0(\bmod 2), \gamma \equiv 1(\bmod 2)$. Hence if $x^{2}=c^{2}$, then $x \in Z(G)$. Now let $X=\{x, y, z\} \subseteq G$. If $X \cap \Omega_{1}(G) \neq \emptyset$, then $X \cap Z(G) \neq \emptyset$ and $\left|X^{2}\right|<9$. Also $\left|X^{2}\right|<9$ if $\left|\left\{x^{2}, y^{2}, z^{2}\right\}\right|<3$. Now assume $x^{2}$, $y^{2}, z^{2}$ pairwise different and not 1 . Then an element of $X$, say $x$, is such that $x^{2}=c^{2}$ and $x \in Z(G)$ by the previous remark. Thus $X \cap Z(G) \neq \emptyset$ and $\left|X^{2}\right|<9$.

Now assume that $G$ satisfies (5). Again we have $\Omega_{1}(G) \leqslant Z(G)$ and $\mho_{1}(G)=$ $\left\{1, a^{2}, b^{2}, c^{2}\right\}$. Moreover every element $g$ of $G$ can be written as $g=f a^{\alpha} b^{\beta} c^{\gamma} d^{\delta}$ where $\alpha, \beta, \gamma, \delta \equiv 0,1(\bmod 2)$. As before it is easy to see that $g^{2}=a^{2}$ if and only if either $\beta \equiv 1(\bmod 2), \gamma \equiv 0(\bmod 2), \delta \equiv 1(\bmod 2)$, that is, $g \in\left\{f b d, f a b d / f \in \Omega_{1}(G)\right\}$ or $\beta \equiv 0(\bmod 2), \alpha \equiv 1(\bmod 2), \gamma \equiv \delta \equiv 0(\bmod 2)$, that is, $g \in\left\{f a / f \in \Omega_{1}(G)\right\}$. However $g^{2}=c^{2}$ or $g^{2}=1$ if and only if $\alpha \equiv \beta \equiv 0(\bmod 2)$. Hence the elements of $G$ whose square is $a^{2}$ commute with the elements whose square is $c^{2}$. Now it is easy to verify that $G \in D S(3)$.

From the results of [1] and from Theorem $\mathrm{A}$ it follows easily that if $G$ is in $D S(3)$, then $G$ is abelian, or $G=A\langle x\rangle$ where $A$ is an abelian subgroup of index 2 and $a^{x}=a^{-1}$ for every $a \in A$, or $G$ is a 2-group of class less than or equal to 2 , exponent 4 , and with $\left|\mho_{1}(G)\right| \leqslant 4$.

Hence any finite group in $D S(3)$ is soluble. But groups in $D S(k)$ for some $k$ are finite-by-abelian-by-finite, so then any group in $D S(3)$ is soluble.

Also we remark that from Theorem $A$ it follows that if $G$ is a non-abelian finite group in $D S(3)$ then $Z(G)$ has exponent at most 4 , and has exponent 2 if (3) holds.

These will be our starting points in the next section.

\section{Arbitrary groups in $D S(3)$}

We prove the following

ThenRem C. Let $G$ be a group. Then $G \in D S(3)$ if and only if one of (1), (2), (3), (4), (5) of Theorem $A$ holds.

Proof: Let $G \in D S(3)$. Then $G$ is soluble and finite-by-abelian-by-finite, by an unpublished result of P. Neumann ([6], see [3] for a proof).

Assume first that $G$ is finitely generated. Then $G$ is polycyclic and thus residually finite, by a theorem of Hirsch (see for example [7, 5.4.17, p.149]). Suppose $G$ is nonabelian. Then there exists a normal subgroup $N$ of $G$ of finite index with $G / N$ non- 
abelian. If for every $x \in G$ we have $x^{2} H \in Z(G / H)$ for any $H$ normal in $G, H \leqslant N$, of finite index, then $G$ is nilpotent of class 2 and $x^{2} \in Z(G)$. In this case $G$ is periodic. For, if $y$ is a torsion-free element of $G$, we have $y^{2} \in Z(G)$. Thus if $M$ is normal in $G$ with $G / M$ finite, $M \leqslant N$ and $y^{4}, y^{8} \notin M$, we get $y^{2} M \in Z(G / M), G / M$ nonabelian, $y^{2} M$ of order different from 2, 4, a contradiction by the remarks at the end of Section 2 . Then $G$ is a periodic finitely generated nilpotent group, so $G$ is finite and has the required structure.

Now assume that there exist $N$ normal in $G$ of finite index and an element $x N \in$ $G / N$ with $x^{2} N \notin Z(G / N)$. Then $G / N=A / N\langle x N\rangle$ with $a^{x} N=a^{-1} N$, and $A / N$ abelian and $x^{2} \in A$ by Theorem $A$. For every normal subgroup $M \leqslant N$ of finite index we have $x^{2} M \notin Z(G / M)$, hence $G / M=B / M\langle y M\rangle, B / M$ abelian, $y^{2} M \in B / M$, $b^{y} M=b^{-1} M$ for every $b \in B$. If either $A \not \leq B$, or $B \leqslant A$, from $G / M=A / M B / M$, with $A / M, B / M$ abelian of index 2 , it follows that $g^{2} M \in A / M \cap B / M \leqslant Z(G / M)$ for every $g \in G$, a contradiction.

Thus $A=B$, and $x M=b y M$, for some $b \in B$. Hence $G / M=A / M\langle x M\rangle$ with $A / M$ abelian and $a a^{x} \in M$ for every $a \in A$. This holds for any normal $M$ of finite index, $M \leqslant N$ with the same element $x$. Then since $G$ is residually finite we get $G=A\langle x\rangle, A$ abelian of index $2, a^{x}=a^{-1}$ for every $a \in A$, as required.

Now assume that $G$ is an arbitrary group. Suppose $G$ is non-abelian and $\left|\mho_{1}(G)\right|>$ 2. Then there exists a finitely generated subgroup $Y$ of $G$ which is non-abelian and with $\left|\mho_{1}(Y)\right|>2$. First assume that $G$ does not have a subgroup of type (4) nor (5). Then for every finitely generated subgroup $F \geqslant Y$ of $G$ we have $F=A_{F}\langle g\rangle$, where $A_{F}$ is abelian, $g^{2} \in A_{F}$ and $a^{g}=a^{-1}$ for every $a \in A_{F}$. Therefore every finitely generated subgroup of $G$ has an abelian subgroup of index at most 2. Then, using Proposition $1 . \mathrm{K} .2$ of $[5, \mathrm{p} .55]$, it is easy to show that $G$ has an abelian subgroup $A$ of index 2 and $G=A\langle x\rangle$. Assume by contradiction that there exists $a \in A$ with $a^{x} \neq a^{-1}$, $a^{x} \neq a$. Then for every finitely generated subgroup $X \geqslant\langle a, x, F\rangle$ we have $X=B\langle y\rangle$, with $B$ abelian of index 2 and $b^{y}=b^{-1}$ for every $b \in B$. If either $B \leqslant X \cap A$ or $B \geqslant X \cap A$, then $B=X \cap A$ and we have $a \in B, x \notin B$ and $x=c y$, with $c \in B$; then $a^{x}=a^{c y}=a^{y}=a^{-1}$, a contradiction. Thus there exists $z \in B-(X \cap A)$ and we have $z=d x$ with $d \in A$. Then $B \cap X \cap A \leqslant C_{G}(d x) \cap C_{G}(d) \leqslant C_{G}(x)$ and from $|X: B \cap X \cap A| \leqslant 4$ we get $\left|X: C_{G}(x)\right| \leqslant 4$. Furthermore there exists $s \in(A \cap X)-B$ and we have $b^{s}=b^{-1}=b$, for every $b \in B \cap A \cap X$; hence $A \cap B \cap X$ has exponent 2 and is in $Z(X)$. We have proved that $X$ is a finite 2-group nilpotent of class 2 and exponent at most 4 and $\left|X: C_{G}(x)\right| \leqslant 4$. This holds for any $X \geqslant\langle Y, a, x\rangle$. We get easily that $A$ has exponent at most 4 and $\left|G: C_{G}(x)\right| \leqslant 4$. Hence we have $G=Z(G) F$ with $F$ finite, $F \geqslant\langle a, x\rangle$ and $Z(G)$ of exponent 2 by the remark at the end of Section 2. Then $G=T \times F$ with $F$ finite and $\exp T=2$. By Theorem A we 
have a contradiction. Then $A=A_{1} \cup C_{A}(x)$, with $A_{1}=\left\{a \in A / a^{x}=a^{-1}\right\}$ a subgroup of $A$, and $A=A_{1}$ since $G$ is not abelian. Therefore (3) holds.

Now assume that there exists a finite subgroup $H$ of $G$ satisfying (4) or (5) of Theorem A. Then $B$ is not abelian, $\left|\mho_{1}(H)\right|=4$ and $B$ does not have the structure in (3). Then any finitely generated subgroup of $G$ containing $H$ satisfies either (4) or (5). It follows easily that $\left|\mho_{1}(G)\right|=4$ and $\Omega_{1}(G) \leqslant Z(G)$. First assume $H=\langle a, b, c, d\rangle$, $|a|=|b|=|c|=|d|=4, a^{b}=a^{-1}, c^{b}=c^{-1}, c^{d}=c^{-1},[a, c]=[a, d]=[b, d]=1$. Then we have $\langle a\rangle$ and $\langle c\rangle$ normal in $G$. Write $D=C_{G}(a) \cap C_{G}(c)$. Then we have $D$ normal in $G$ and $G=D\langle b, d\rangle$. Furthermore for any $g \in D,|g|=4$, we have $g^{2}=a^{2}$, $g^{2}=c^{2}$ or $g^{2}=b^{2}=(a c)^{2}$. Then $a g, c g$ or $g a c$ has order 2 . Then $D \leqslant \Omega_{1}(G)\langle a, c\rangle$ and $G=\Omega_{1}(G)\langle a, b, c, d\rangle=Y \times\langle a, b, c, d\rangle$ with $Y$ of exponent 2. Therefore (5) holds. Similarly if $G \geqslant\langle a, b, c\rangle$ and $\langle a, b, c\rangle$ satisfies (4) of Theorem A, then we get $G=Y \times\langle a, b, c\rangle$, with $\exp Y=2$, and (4) holds.

\section{References}

[1] J.G. Berkovich, G.A. Freiman and C.E. Praeger, 'Small squaring and cubing properties of finite groups', Bull. Austral. Math. Soc. 44 (1991), 429-450.

[2] G.A. Freiman, 'On two and three-element subsets of groups', Aequationes Math. 22 (1981), 140-152.

[3] M. Herzog, P. Longobardi and M. Maj, 'On a combinatorial problem in group theory', submitted.

[4] B. Huppert, Endliche Gruppen I (Springer-Verlag, Berlin, Heidelberg, New York, 1967).

[5] O.H. Kegel and B.A.F. Wehrfritz, Locally finite groups (North-Holland Publishing Company, Amsterdam, London, 1973).

[6] P.M. Neumann, 'A combinatorial problem in group theory', Canberra 1989, private communication.

[7] D.J.S. Robinson, A course in the theory of groups (Springer-Verlag, Berlin, Heidelberg, New York, 1982).

Dip di Matematica e Applicazioni

Università degli Studi di Napoli

Monte S. Angelo, via Cinthia

80126 Naples

Italy 